\title{
Embedding of analytic function spaces with given mean growth of the derivative
}

\author{
Óscar Blasco*1, Daniel Girela*2 ${ }^{* * 2}$, and M. Auxiliadora Márquez ${ }^{* * 2}$ \\ ${ }^{1}$ Departamento de Análisis Matemático, Facultad de Ciencias Matemáticas, Universidad de Valencia, 46100 Burjasot, Spain \\ ${ }^{2}$ Departamento de Análisis Matemático, Facultad de Ciencias, Universidad de Málaga, 29071 Málaga, Spain
}

Key words Integral means, Hardy spaces, Banach envelopes, embedding theorems, Dini weights, $b_{q}$-weights. MSC (2000) 30D55

If $\phi$ is a positive function defined in $[0,1)$ and $0<p<\infty$, we consider the space $\mathcal{L}(p, \phi)$ which consists of all functions $f$ analytic in the unit disc $\mathbb{D}$ for which the integral means of the derivative $M_{p}\left(r, f^{\prime}\right)=$ $\left(\frac{1}{2 \pi} \int_{-\pi}^{\pi}\left|f^{\prime}\left(r e^{i \theta}\right)\right|^{p} d \theta\right)^{1 / p}, 0<r<1$, satisfy $M_{p}\left(r, f^{\prime}\right)=\mathrm{O}(\phi(r))$, as $r \rightarrow 1$. In this paper, for any given $p \in(0,1)$, we characterize the functions $\phi$, among a certain class of weight functions, to be able to embedd $\mathcal{L}(p, \phi)$ into classical function spaces. These results complement other previously obtained by the authors for $p \geq 1$.

Copyright line will be provided by the publisher

\section{Introduction.}

Let $\mathbb{D}$ denote the unit disc $\{z \in \mathbb{C}:|z|<1\}$. If $0<r<1$ and $g$ is a function which is analytic in $\mathbb{D}$, we set

$$
\begin{gathered}
M_{p}(r, g)=\left(\frac{1}{2 \pi} \int_{-\pi}^{\pi}\left|g\left(r e^{i \theta}\right)\right|^{p} d \theta\right)^{1 / p}, \quad 0<p<\infty \\
M_{\infty}(r, g)=\max _{|z|=r}|g(z)| .
\end{gathered}
$$

For $0<p \leq \infty$ the Hardy space $H^{p}$ consists of those functions $g$, analytic in $\mathbb{D}$, for which

$$
\|g\|_{H^{p}}=\sup _{0<r<1} M_{p}(r, g)<\infty .
$$

We refer to [8] and [9] for the theory of Hardy spaces.

In this paper we shall be dealing with functions $f$, analytic in $\mathbb{D}$, for which the integral means $M_{p}\left(r, f^{\prime}\right)$ are dominated by a certain weight function $\phi(r)$. Namely, if $0<p \leq \infty$ and $\phi$ is a non-negative function defined in $[0,1)$, we define $\mathcal{L}(p, \phi)$ as the space of all functions $f$ which are analytic in $\mathbb{D}$ and satisfy

$$
M_{p}\left(r, f^{\prime}\right)=\mathrm{O}(\phi(r)), \quad \text { as } r \rightarrow 1 .
$$

For $1 \leq p \leq \infty$, these spaces were extensively studied by the authors in [4]. In this paper we will focus our attention in the other values of $p, 0<p<1$. Our aim is characterizing those "natural" functions $\phi$ for which the space $\mathcal{L}(p, \phi)$ is embedded into classical function spaces.

A well known result of Hardy and Littlewood (see Theorem 5.12 of [8]) asserts that if $0<p<1$ and $\phi$ is bounded then $\mathcal{L}(p, \phi) \subset H^{q}$, where $q=p /(1-p)$. Moreover, for each value of $p$, the exponent $q$ is best possible. Consequently, we shall not consider this case. Also, we shall assume without loss of generality that the function $\phi$ is increasing and continuous. Certainly, some restriction on the growth of $\phi$ is needed if we expect to

\footnotetext{
* oblasco@uv.es

** Corresponding author: e-mail:girela@uma.es, Phone: +00 34 952131905, Fax: +0034952131894

***marquez@anamat.cie.uma.es
} 
be able to embed the space $\mathcal{L}(p, \phi)$ into some classical spaces. We shall assume that $\phi \in L^{1}((0,1))$. This and the fact that $\phi$ is increasing imply that

$$
(1-r) \phi(r) \rightarrow 0, \quad \text { as } r \rightarrow 1 .
$$

Because of some technical reasons we shall also assume that the function $r \mapsto(1-r) \phi(r)$ is decreasing in $[0,1)$.

Summarizing, throughout this paper we shall consider the class $\mathcal{A F}$ which consists of those functions $\phi:[0,1) \rightarrow[0, \infty)$ which are increasing and continuous, and satisfy

$$
\lim _{r \rightarrow 1} \phi(r)=\infty, \quad \phi \in L^{1}((0,1)) \quad \text { and } \quad(1-r) \phi(r) \downarrow, \text { as } r \uparrow 1 .
$$

The elements of $\mathcal{A F}$ will be called admissible functions. Using the fact that $(1-r) \phi(r)$ is a decreasing function, we readily obtain the following result.

Lemma 1.1 If $\phi \in \mathcal{A F}$ then $\phi\left(\frac{1+r}{2}\right) \leq 2 \phi(r)$, for all $r \in(0,1)$.

\section{Embedding $\mathcal{L}(\mathbf{p}, \phi)$ into $\mathbf{B}^{\mathrm{p}}$.}

It is easy to check (see e. g. p. 898 of [4]) that,

$$
\text { if } p \geq 1 \text { and } \phi \in \mathcal{A F} \text {, then } \mathcal{L}(p, \phi) \subset H^{p} \text {. }
$$

This is not true for $p<1$. Indeed, if $0<p<1$ and

$$
f(z)=\frac{1}{(1-z)^{1 / p}\left(\log \frac{2}{1-z}\right)^{1 / p}}, \quad z \in \mathbb{D},
$$

then it is not difficult to prove (use for instance the asymptotic expressions (2.33) in p. 192 of Vol. I of [18]) that $f \in \mathcal{L}(p, \phi)$ with

$$
\phi(r)=\frac{1}{(1-r)\left(\log \frac{2}{1-r}\right)^{1 / p}}, \quad r_{0}<r<1 .
$$

(hence $\phi \in \mathcal{A F}$ ) but $f \notin H^{p}$.

For $0<p<1$, let $B^{p}$ be the "containing Banach space" or "Banach envelope" of $H^{p}$, that is, the space of all analytic functions $f$ in $\mathbb{D}$ such that

$$
\int_{0}^{1}(1-r)^{\frac{1}{p}-2} M_{1}(r, f) d r<\infty .
$$

By Theorem 5.11 of [8] with $q=\lambda=1$, we see that $H^{p} \subset B^{p}$ for all $p \in(0,1)$, a result which was originally proved by Hardy and Littlewood ([11], p. 412). Duren, Romberg and Shields [7] proved that $H^{p}$ is dense in $B^{p}$ and that the two spaces have the same continuous linear functionals. Our first result is a substitute of (2) for $p<1$.

Theorem 2.1 If $0<p<1$ and $\phi \in \mathcal{A F}$, then $\mathcal{L}(p, \phi) \subset B^{p}$.

Proof. Take $f \in \mathcal{L}(p, \phi)$. Adding a positive constant to $\phi$ if necessary, we may assume that $M_{p}\left(r, f^{\prime}\right) \leq$ $K \phi(r)$ for all $r \in(0,1)$. Using Theorem 5.11 of [8] with $q=\lambda=1$, we obtain

$$
\int_{0}^{1}(1-r)^{\frac{1}{p}-2} M_{1}\left(r s, f^{\prime}\right) d r \leq K M_{p}\left(s, f^{\prime}\right) \leq K \phi(s), \quad 0<s<1 .
$$

Now,

$$
\left|f\left(r e^{i \theta}\right)\right| \leq|f(0)|+\int_{0}^{1}\left|f^{\prime}\left(s r e^{i \theta}\right)\right| d s, \quad \theta \in \mathbb{R}, \quad 0<r<1,
$$


and, hence,

$$
M_{1}(r, f) \leq|f(0)|+\int_{0}^{1} M_{1}\left(r s, f^{\prime}\right) d s, \quad 0<r<1 .
$$

Then, using (4), Fubini's theorem, (3) and the fact that $\phi \in L^{1}((0,1))$, we obtain

$$
\begin{aligned}
& \int_{0}^{1}(1-r)^{\frac{1}{p}-2} M_{1}(r, f) d r \leq K_{1}+\int_{0}^{1}(1-r)^{\frac{1}{p}-2} \int_{0}^{1} M_{1}\left(r s, f^{\prime}\right) d s d r \\
= & K_{1}+\int_{0}^{1} \int_{0}^{1}(1-r)^{\frac{1}{p}-2} M_{1}\left(r s, f^{\prime}\right) d r d s \leq K_{1}+K_{2} \int_{0}^{1} \phi(s) d s<\infty .
\end{aligned}
$$

Hence, $f \in B^{p}$.

Let us remark that here and all over the paper we shall be using the convention that $K, K_{1}, \ldots$, denote positive constants (which may depend upon $p, q, \phi, \omega, f, \ldots$ but not on $r, t, s$, or $n$ ), not necessarily the same at different occurrences.

\section{Embedding $\mathcal{L}(\mathbf{p}, \phi)$ into $\mathbf{H}^{\mathrm{q}}, \mathbf{p} \leq \mathbf{q}<\infty$.}

We start this section finding, for any given $q \in[p, \infty)$, a condition on $\phi$ which implies that $\mathcal{L}(p, \phi) \subset H^{q}$.

Theorem 3.1 Suppose that $0<p<1$ and $\phi \in \mathcal{A F}$. If $p \leq q<\infty$ and $\phi(r)(1-r)^{1-\frac{1}{p}} \in L^{q}((0,1))$, then $\mathcal{L}(p, \phi) \subset H^{q}$.

Before embarking into the proof, let us introduce another family of spaces. For $0<p<\infty$, the space of Dirichlet type $\mathcal{D}_{p-1}^{p}$ consists of all functions $f$ which are analytic in $\mathbb{D}$ and satisfy

$$
\int_{\mathbb{D}}\left(1-|z|^{2}\right)^{p-1}\left|f^{\prime}(z)\right|^{p} d x d y<\infty .
$$

The spaces $\mathcal{D}_{p-1}^{p}$ are closely related to Hardy spaces. Indeed, a direct calculation with power series shows that $H^{2}=\mathcal{D}_{1}^{2}$. A classical result of Littlewood and Paley [14] (see also [15]) asserts that if $2 \leq p<\infty$ then $H^{p} \subset \mathcal{D}_{p-1}^{p}$. This result can be proved by Riesz-Thorin interpolation. On the other hand, we have

$$
\mathcal{D}_{p-1}^{p} \subset H^{p}, \quad 0<p \leq 2 .
$$

For $1<p<2$ this inclusion can also be proved by interpolation, since the inclusion $\mathcal{D}_{0}^{1} \subset H^{1}$ is trivial. Vinogradov ([16], Lemma 1.4) gave a proof of the inclusion for $0<p<1$.

Our proof of Theorem 3.1 will be based on (5). Actually, we shall use the following result which follows from (5) using the closed graph theorem.

Proposition A If $0<p \leq 2$ then there exists a positive constant $K_{p}$, which only depends on $p$, such that

$$
\|f\|_{H^{p}}^{p} \leq K_{p}\left(|f(0)|^{p}+\int_{\mathbb{D}}\left(1-|z|^{2}\right)^{p-1}\left|f^{\prime}(z)\right|^{p} d x d y\right)
$$

for every $f \in \mathcal{D}_{p-1}^{p}$.

We shall use also the following result, due essentially to Hardy and Littlewood, which can be proved by modifying the proof of Theorem 5.9 in [8].

Proposition B For $0<p<q \leq \infty$, there exists a constant $K_{p, q}$, depending only on $p$ and $q$, such that for each analytic function $f$ in $\mathbb{D}$ and each $r \in(0,1)$ we have

$$
M_{q}(r, f) \leq K_{p, q} M_{p}\left(\frac{1+r}{2}, f\right)(1-r)^{\frac{1}{q}-\frac{1}{p}} .
$$


Proof of Theorem 3.1. Take $f \in \mathcal{L}(p, \phi)$. Assume, without loss of generality, that $M_{p}\left(r, f^{\prime}\right) \leq K \phi(r)$, $0<r<1$.

Suppose first that $p \leq q \leq 2$. Using Proposition A, Proposition B, the fact that $f \in \mathcal{L}(p, \phi)$, Lemma 1.1 and the monotony of the function $\phi$ and of the function $t \mapsto 1 /(1-t)$ we deduce that

$$
\begin{aligned}
M_{q}(r, f)^{q} & \leq K\left(|f(0)|^{q}+\int_{0}^{1}(1-s)^{q-1} M_{q}\left(r s, f^{\prime}\right)^{q} d s\right) \\
& \leq K\left(|f(0)|^{q}+\int_{0}^{1}(1-s)^{q-1}\left(\frac{M_{p}\left(\frac{1+r s}{2}, f^{\prime}\right)}{(1-r s)^{\frac{1}{p}-\frac{1}{q}}}\right)^{q} d s\right) \\
& \leq K\left(|f(0)|^{q}+\int_{0}^{1}(1-s)^{q-1}\left(\frac{\phi\left(\frac{1+r s}{2}\right)}{(1-r s)^{\frac{1}{p}-\frac{1}{q}}}\right)^{q} d s\right) \\
& \leq K\left(|f(0)|^{q}+\int_{0}^{1}(1-s)^{q-1}\left(\frac{\phi(r s)}{(1-r s)^{\frac{1}{p}-\frac{1}{q}}}\right)^{q} d s\right) \\
& \leq K\left(|f(0)|^{q}+\int_{0}^{1}(1-s)^{q-1}\left(\frac{\phi(s)}{(1-s)^{\frac{1}{p}-\frac{1}{q}}}\right)^{q} d s\right) \\
& =K\left(|f(0)|^{q}+\int_{0}^{1}\left((1-s)^{1-\frac{1}{p}} \phi(s)\right)^{q} d s\right), \quad 0<r<1 .
\end{aligned}
$$

Since $\phi(r)(1-r)^{1-\frac{1}{p}} \in L^{q}((0,1))$, we deduce that $\sup _{0<r<1} M_{q}(r, f)<\infty$, that is, $f \in H^{q}$.

To deal with the case $2<q<\infty$ we use duality. Let $q^{\prime}$ be the exponent conjugate of $q$, that is, $\frac{1}{q}+\frac{1}{q^{\prime}}=1$. Take a polynomial $g$. Taking $q=\infty$ and $p=\lambda=q^{\prime}$ in Theorem 5.11 of [8] and using the closed graph theorem, we see that

$$
\left(\int_{0}^{1} M_{\infty}(r, g)^{q^{\prime}} d r\right)^{1 / q^{\prime}} \leq C\|g\|_{H^{q^{\prime}}}
$$

Since $2<q$, we have that $\phi(r)(1-r)^{1-\frac{1}{p}} \in L^{2}((0,1))$ and then, using the case just proved, we deduce that $f \in H^{2}$. Hence, $f$ has a finite radial limit $f\left(e^{i \theta}\right)$ for almost every $\theta$. We have,

$$
\begin{aligned}
\left|\int_{-\pi}^{\pi}\left(f\left(e^{i \theta}\right)-f(0)\right) \bar{g}\left(e^{i \theta}\right) \frac{d \theta}{2 \pi}\right| & =2\left|\int_{0}^{1} \int_{-\pi}^{\pi} f^{\prime}\left(r e^{i \theta}\right) \bar{g}\left(r e^{i \theta}\right) e^{i \theta} \frac{d \theta}{2 \pi} d r\right| \\
& \leq 2 \int_{0}^{1} M_{1}\left(r, f^{\prime}\right) M_{\infty}(r, g) d r .
\end{aligned}
$$

Then, using Proposition B and Lemma 1.1 as above, the fact that $f \in \mathcal{L}(p, \phi)$, Hölder's inequality, (9) and the hypothesis $\frac{\phi(r)}{(1-r)^{\frac{1}{p}-1}} \in L^{q}((0,1))$, we obtain

$$
\begin{aligned}
& \left|\int_{-\pi}^{\pi}\left(f\left(e^{i \theta}\right)-f(0)\right) \bar{g}\left(e^{i \theta}\right) \frac{d \theta}{2 \pi}\right| \\
\leq & K \int_{0}^{1} \frac{M_{p}\left(r, f^{\prime}\right)}{(1-r)^{\frac{1}{p}-1}} M_{\infty}(r, g) d r \\
\leq & K \int_{0}^{1} \frac{\phi(r)}{(1-r)^{\frac{1}{p}-1}} M_{\infty}(r, g) d r \\
\leq & K\left(\int_{0}^{1}\left(\frac{\phi(r)}{(1-r)^{\frac{1}{p}-1}}\right)^{q} d r\right)^{1 / q}\left(\int_{0}^{1} M_{\infty}(r, g)^{q^{\prime}} d r\right)^{1 / q^{\prime}} \\
\leq & K\left(\int_{0}^{1}\left(\frac{\phi(r)}{(1-r)^{\frac{1}{p}-1}}\right)^{q} d r\right)^{1 / q}\|g\|_{H^{q^{\prime}}},
\end{aligned}
$$


with $K$ independent of $g$. By duality, this shows that $f \in H^{q}$. This finishes the proof for all values of $q$.

Our next goal is to prove that the converse of Theorem 3.1 is true if we impose to the admissible function $\phi$ a certain natural regularity condition.

Definition 3.2 Given $\phi \in \mathcal{A F}$, we define $\omega_{\phi}:[0,1) \rightarrow[0, \infty)$ by $\omega_{\phi}(0)=0$ and $\omega_{\phi}(t)=t \phi(1-t)$, $0<t<1$. Hence,

$$
\phi(r)=\frac{\omega_{\phi}(1-r)}{1-r}, \quad 0<r<1 .
$$

Recall that if $\phi \in \mathcal{A F}$ then $(1-r) \phi(r) \downarrow 0$, as $r \uparrow 1$. Then it follows easily that $\omega_{\phi}$ is continuous and increasing in $[0,1)$.

Definition 3.3 Let $\omega:[0,1) \rightarrow[0, \infty)$ be a continuous function with $\omega(0)=0$.

(i) We say that $\omega$ satisfies the Dini condition, or that $\omega$ is a Dini weight, if there exists a positive constant $K$ such that

$$
\int_{0}^{s} \frac{\omega(t)}{t} d t \leq K \omega(s), \quad 0<s<1
$$

(ii) If $\alpha>0$, we say that $\omega$ satisfies the condition $b_{\alpha}$, or that $\omega$ is a $b_{\alpha}$-weight, if there exists a positive constant $K$ such that

$$
\int_{s}^{1} \frac{\omega(t)}{t^{\alpha+1}} d t \leq K \frac{\omega(s)}{s^{\alpha}}, \quad 0<s<1
$$

Dini weights and $b_{\alpha}$-weights show up in a natural way in many questions concerning spaces of analytic functions (see, e. g., [2], [3], [4], [5], [6], [10], [13]).

Definition 3.4 For $0<p \leq 1$, we shall say that a function $\phi \in \mathcal{A F}$ satisfies the condition $C_{p}$ if $\omega_{\phi}^{p}$ satisfies the Dini condition and the condition $b_{p}$.

Interesting examples of admissible functions which satisfy the condition $C_{p}$ are given next (see Proposition 1.2 of [2]).

Example 3.5 If $0<\alpha<1, \beta>0$ and $\phi(r)=\frac{1}{(1-r)^{\alpha}}\left(\log \frac{e}{1-r}\right)^{\beta},\left(r_{0}<r<1\right)$ then $\phi \in \mathcal{A F}$ and satisfies the condition $C_{p}$ for every $p \in(0,1)$.

We shall prove the following result.

Theorem 3.6 Suppose that $0<p<1, p \leq q<\infty$ and $\phi \in \mathcal{A F}$. If $\phi$ satisfies the condition $C_{p}$ then the following statements are equivalent:

(i) $\mathcal{L}(p, \phi) \subset H^{q}$.

(ii) $\phi(r)(1-r)^{1-\frac{1}{p}} \in L^{q}((0,1))$.

The implication (ii) $\Rightarrow$ (i) follows from Theorem 3.1. Several definitions and results will be needed to prove the other implication.

Definition 3.7 If $0<p \leq 1$ and $\phi \in \mathcal{A F}$, we define

$$
G_{p, \phi}(z)=\sum_{j=1}^{\infty} \frac{\omega_{\phi}\left(\delta_{j}\right)}{\left(1-z+\delta_{j}\right)^{1 / p}}, \quad z \in \mathbb{D},
$$

where, $\delta_{j}=2^{-j}, j \geq 0$. Define also

$$
\Phi_{p}(r)=\left(\int_{0}^{1} \frac{\omega_{\phi}^{p}(t)}{(1-r+t)^{p}} \frac{d t}{t}\right)^{1 / p}, \quad 0<r<1 .
$$


Notice that the series in (14) converges uniformly on each compact subset of $\mathbb{D}$ and, therefore, it defines a function which is analytic in $\mathbb{D}$.

Now, we have

$$
\phi(r) \leq K \Phi_{p}(r), \quad 0<r<1
$$

Indeed, bearing in mind that $\phi$ is increasing, the definitions and the fact that $p \leq 1$, we have

$$
\begin{aligned}
\phi(r) & \leq\left(\frac{2}{1-r} \int_{r}^{\frac{1+r}{2}} \phi(t)^{p} d t\right)^{1 / p}=\left(\frac{2}{1-r} \int_{\frac{1-r}{2}}^{1-r} \phi(1-t)^{p} d t\right)^{1 / p} \\
& =\left(\frac{2}{1-r} \int_{\frac{1-r}{2}}^{1-r} \frac{\omega_{\phi}(t)^{p}}{t^{p}} d t\right)^{1 / p} \leq K\left(\int_{\frac{1-r}{2}}^{1-r} \frac{\omega_{\phi}(t)^{p}}{t^{p}} \frac{d t}{t}\right)^{1 / p} \leq K \Phi_{p}(r) .
\end{aligned}
$$

The other inequality holds if and only if $\phi$ satisfies the condition $C_{p}$ :

Proposition 3.8 Let $0<p \leq 1$ and $\phi \in \mathcal{A F}$. The following statements are equivalent:

(i) $\phi$ satisfies the condition $C_{p}$.

(ii) There exists a positive constant $K$ such that

$$
\Phi_{p}(r) \leq K \phi(r), \quad 0<r<1
$$

Proof. For simplicity, write $\omega$ for $\omega_{\phi}$. Notice that $2(1-r) \geq(1-r+t)$, if $0<t \leq 1-r$, and then

$$
\frac{1}{(1-r)^{p}} \int_{0}^{1-r} \frac{\omega(t)^{p}}{t} d t \leq K \int_{0}^{1-r} \frac{\omega(t)^{p}}{t(1-r+t)^{p}} d t \leq K \Phi_{p}^{p}(r), \quad 0<r<1 .
$$

Also, since $1-r+t \leq 2 t$ whenever $1-r \leq t<1$, we have

$$
\int_{1-r}^{1} \frac{\omega(t)^{p}}{t^{p+1}} d t \leq K \int_{1-r}^{1} \frac{\omega(t)^{p}}{t(1-r+t)^{p}} d t \leq K \Phi_{p}^{p}(r), \quad 0<r<1 .
$$

Using (18) and (19), we easily deduce that (ii) $\Rightarrow$ (i).

The implication (i) $\Rightarrow$ (ii) follows using that

$$
\Phi_{p}^{p}(r) \leq \frac{1}{(1-r)^{p}} \int_{0}^{1-r} \frac{\omega^{p}(t)}{t} d t+\int_{1-r}^{1} \frac{\omega^{p}(t)}{t^{p+1}} d t .
$$

Next, for an admissible function $\phi$ satisfying the condition $C_{p}$, we shall prove that the function $G_{p, \phi}$ belongs to the space $\mathcal{L}(p, \phi)$. This fact will be basic to prove the implication (i) $\Rightarrow$ (ii) in Theorem 3.6.

Proposition 3.9 Let $0<p \leq 1$ and $\phi \in \mathcal{A F}$. If $\phi$ satisfies the condition $C_{p}$ then $G_{p, \phi} \in \mathcal{L}(p, \phi)$.

Proof. Write $\omega$ for $\omega_{\phi}$. Then,

$$
G_{p, \phi}^{\prime}(z)=\frac{1}{p} \sum_{j=1}^{\infty} \frac{\omega\left(\delta_{j}\right)}{\left(1-z+\delta_{j}\right)^{1+\frac{1}{p}}}, \quad z \in \mathbb{D}
$$


and, hence we have, for $0<r<1$,

$$
\begin{aligned}
& M_{p}^{p}\left(G_{p, \phi}^{\prime}\right) \leq K \sum_{j=1}^{\infty} \omega^{p}\left(\delta_{j}\right) \int_{-\pi}^{\pi} \frac{d \theta}{\mid 1+\delta_{j}-r e^{i \theta \mid p+1}} \\
\leq & K \sum_{j=1}^{\infty} \omega^{p}\left(\delta_{j}\right) \int_{-\pi}^{\pi} \frac{d \theta}{\left|1-\frac{r}{1+\delta_{j}} e^{i \theta}\right| p+1} \leq K \sum_{j=1}^{\infty} \omega^{p}\left(\delta_{j}\right) \frac{1}{\left|1-\frac{r}{1+\delta_{j}}\right|^{p}} \\
\leq & K \sum_{j=1}^{\infty} \frac{\omega^{p}\left(\delta_{j}\right)}{\left(1+\delta_{j}-r\right)^{p}} \leq K \sum_{j=1}^{\infty} \frac{\omega^{p}\left(\delta_{j}\right)\left(\delta_{j}-\delta_{j+1}\right)}{\delta_{j}\left(1+\delta_{j}-r\right)^{p}} .
\end{aligned}
$$

Now,

$$
\frac{1}{\delta_{j}^{1-p}\left(1+\delta_{j}-r\right)^{p}} \leq \frac{1}{t^{1-p}(1+t-r)^{p}}, \quad t \in\left[\delta_{j+1}, \delta_{j}\right]
$$

and, since $\frac{\omega(t)}{t}$ is decreasing,

$$
\frac{\omega^{p}\left(\delta_{j}\right)}{\delta_{j}^{p}} \leq \frac{\omega^{p}(t)}{t^{p}}, \quad t \in\left[\delta_{j+1}, \delta_{j}\right]
$$

Then

$$
\frac{\omega^{p}\left(\delta_{j}\right)\left(\delta_{j}-\delta_{j+1}\right)}{\delta_{j}\left(1+\delta_{j}-r\right)^{p}} \leq K \int_{\delta_{j+1}}^{\delta_{j}} \frac{\omega^{p}(t)}{t(1+t-r)^{p}} d t, \quad j \geq 1
$$

which, using (20) and Proposition 3.8, implies

$$
M_{p}^{p}\left(r, G_{p, \phi}^{\prime}\right) \leq K \int_{0}^{1} \frac{\omega^{p}(t)}{t(1+t-r)^{p}} d t=K \Phi_{p}^{p}(r) \leq K \phi^{p}(r), \quad 0<r<1 .
$$

Hence, $G_{p, \phi} \in \mathcal{L}(p, \phi)$.

Proof of Theorem 3.6. It only remains to prove the implication (i) $\Rightarrow$ (ii). So, suppose that $0<p<1$, $p \leq q<\infty, \phi \in \mathcal{A F}$ satisfies the condition $C_{p}$ and $\mathcal{L}(p, \phi) \subset H^{q}$. For simplicity, write $\omega$ for $\omega_{\phi}$ and $G$ for $G_{p, \phi}$.

Since $\phi$ satisfies the condition $C_{p}$, using Proposition 3.9 we deduce that $G \in H^{q}$ and then, using the FejérRiesz inequality (see Theorem 3.13 of [8]) we obtain

$$
\int_{0}^{1} G(r)^{q} d r<\infty
$$

Now, bearing in mind the definition of $\delta_{j}$ and the fact the function $t \mapsto \frac{\omega(t)}{t}$ is decreasing, we see that

$$
G(r)=\sum_{j=1}^{\infty} \frac{\omega\left(\delta_{j}\right)}{\left(1-r+\delta_{j}\right)^{1 / p}}=\sum_{j=1}^{\infty} \frac{\omega\left(\delta_{j}\right)\left(\delta_{j-1}-\delta_{j}\right)}{\delta_{j}\left(1-r+\delta_{j}\right)^{1 / p}} \geq \int_{0}^{1} \frac{\omega(t)}{(1+t-r)^{1 / p}} \frac{d t}{t}, \quad 0<r<1
$$

Then, using that $\omega$ is increasing, we have

$$
\begin{aligned}
& \int_{0}^{1} G(r)^{q} d r \geq \int_{0}^{1}\left(\int_{0}^{1} \frac{\omega(t)}{(1+t-r)^{1 / p}} \frac{d t}{t}\right)^{q} d r \\
= & \int_{0}^{1}\left(\int_{0}^{1} \frac{\omega(t)}{t(s+t)^{1 / p}} d t\right)^{q} d s \geq \int_{0}^{1}\left(\int_{s}^{1} \frac{\omega(t)}{t(s+t)^{1 / p}} d t\right)^{q} d s \\
\geq & K \int_{0}^{1} \omega(s)^{q}\left(\int_{s}^{1} \frac{1}{t^{\frac{1}{p}+1}} d t\right)^{q} d s=K \int_{0}^{1} \omega(s)^{q}\left(\frac{1}{s^{1 / p}}-1\right)^{q} d s
\end{aligned}
$$


which, together with (21), shows that $\omega(s)\left(\frac{1}{s^{1 / p}}-1\right) \in L^{q}((0,1))$. Clearly, this implies that $\frac{\omega(s)}{s^{1 / p}} \in L^{q}((0,1))$ or, equivalently, that $\phi(r)(1-r)^{1-\frac{1}{p}} \in L^{q}((0,1))$.

Let us close this section noticing that the condition $q<\infty$ is needed in Theorem 3.1 and in Theorem 3.6. Indeed, we have already remarked that if $0<p<1$ and $\epsilon>0$ then there exists a function $f$ which is analytic in $\mathbb{D}$ with $f^{\prime} \in H^{p}$ such that $f \notin H^{\frac{p}{1-p}+\epsilon}$ (hence, $f \notin H^{\infty}$ ). Actually, we can say even more.

Recall that if $f$ is an analytic function in $\mathbb{D}$ then $f$ is said to be a Bloch function if

$$
\sup _{z \in \mathbb{D}}\left(1-|z|^{2}\right)\left|f^{\prime}(z)\right|<\infty
$$

and it is said to be a normal function in the sense of Lehto and Virtanen [12] if

$$
\sup _{z \in \mathbb{D}}\left(1-|z|^{2}\right) \frac{\left|f^{\prime}(z)\right|}{1+|f(z)|^{2}}<\infty
$$

The space of all Bloch functions will be denoted by $\mathcal{B}$ and $\mathcal{N}$ will stand for the class of all normal analytic functions in $\mathbb{D}$. We refer to [1] for the theory of Bloch functions and normal functions. Notice that $H^{\infty} \subset \mathcal{B} \subset$ $\mathcal{N}$. Using Theorem 5.9 of [8] we see that for any $p \in(0,1)$ there exists a function $f$ which is analytic in $\mathbb{D}$ with $f^{\prime} \in H^{p}$ but $f \notin \mathcal{B}$. A result of Yamashita [17] implies the following stronger result which should be compared with Theorem 1.3 of [4].

Theorem A There exists a function $f$ which is analytic in $\mathbb{D}$ with $f^{\prime} \in H^{p}$ for all $p \in(0,1)$ but such that $f$ is not a normal function.

\section{Embedding $\mathcal{L}(\mathrm{p}, \phi)$ into $\mathrm{B}^{\mathrm{q}}, \mathrm{p}<\mathrm{q}<1$.}

We can prove the following result.

Theorem 4.1 Suppose that $0<p<q<1$ and $\phi \in \mathcal{A F}$. If $\phi$ satisfies that $\phi(r)(1-r)^{\frac{1}{q}-\frac{1}{p}} \in L^{1}((0,1))$, then $\mathcal{L}(p, \phi) \subset B^{q}$.

The proof of Theorem 4.1 follows the lines of that of Theorem 2.1, taking into account Proposition B. We omit the details.

Next we shall see that the converse is true if $\phi$ satisfies the condition $C_{p}$.

Theorem 4.2 Suppose that $0<p<q<1$ and $\phi \in \mathcal{A F}$. If $\phi$ satisfies the condition $C_{p}$ then the following statements are equivalent:

(i) $\mathcal{L}(p, \phi) \subset B^{q}$.

(ii) $\phi(r)(1-r)^{\frac{1}{q}-\frac{1}{p}} \in L^{1}((0,1))$.

Pro of. We only have to prove the implication (i) $\Rightarrow$ (ii). So, assume (i). Once more, write $\omega$ for $\omega_{\phi}$ and $G$ for $G_{p, \phi}$.

Using Proposition 3.9, we deduce that $G \in B^{q}$.

Observe that, if $(1-z)^{\frac{-1}{p}}=\sum_{n=0}^{\infty} \gamma_{n} z^{n}$, then

$$
G(r z)=\sum_{n=0}^{\infty} \gamma_{n} r^{n}\left(\sum_{j=1}^{\infty} \frac{\omega\left(\delta_{j}\right)}{\left(1+\delta_{j}\right)^{\frac{1}{p}+n}}\right) z^{n}, \quad 0<r<1, \quad|z| \leq 1,
$$

and then, using Hardy's inequality (see p. 48 of [8]), we obtain

$$
\sum_{n=0}^{\infty} \frac{\gamma_{n}}{n+1} r^{n}\left(\sum_{j=1}^{\infty} \frac{\omega\left(\delta_{j}\right)}{\left(1+\delta_{j}\right)^{\frac{1}{p}+n}}\right) \leq K M_{1}(r, G), \quad 0<r<1 .
$$


Notice that

$$
\sum_{n=0}^{\infty} \frac{\gamma_{n}}{n+1} r^{n}\left(\sum_{j=1}^{\infty} \frac{\omega\left(\delta_{j}\right)}{\left(1+\delta_{j}\right)^{\frac{1}{p}+n}}\right)=\sum_{j=1}^{\infty} \frac{\omega\left(\delta_{j}\right)}{\left(1+\delta_{j}\right)^{\frac{1}{p}}}\left(\sum_{n=0}^{\infty} \frac{\gamma_{n}}{n+1}\left(\frac{r}{1+\delta_{j}}\right)^{n}\right) .
$$

Now, since $\gamma_{n} \approx(n+1)^{\frac{1}{p}-1}$, we see that

$$
\sum_{n=0}^{\infty} \frac{\gamma_{n}}{n+1}\left(\frac{r}{1+\delta_{j}}\right)^{n} \approx\left(1-\frac{r}{1+\delta_{j}}\right)^{1-\frac{1}{p}} \approx\left(1+\delta_{j}-r\right)^{1-\frac{1}{p}}
$$

and then, using (24), we obtain

$$
\sum_{n=0}^{\infty} \frac{\gamma_{n}}{n+1} r^{n}\left(\sum_{j=1}^{\infty} \frac{\omega\left(\delta_{j}\right)}{\left(1+\delta_{j}\right)^{\frac{1}{p}+n}}\right) \approx \sum_{j=1}^{\infty} \frac{\omega\left(\delta_{j}\right)}{\left(1+\delta_{j}-r\right)^{\frac{1}{p}-1}}
$$

which, together with (23), implies

$$
\sum_{j=1}^{\infty} \frac{\omega\left(\delta_{j}\right)}{\left(1+\delta_{j}-r\right)^{\frac{1}{p}-1}} \leq K M_{1}(r, G), \quad 0<r<1 .
$$

Since $G \in B^{q},(25)$ implies

$$
\int_{0}^{1}(1-r)^{\frac{1}{q}-2}\left(\sum_{j=1}^{\infty} \frac{\omega\left(\delta_{j}\right)}{\left(1+\delta_{j}-r\right)^{\frac{1}{p}-1}}\right) d r<\infty
$$

Bearing in mind that $\delta_{j}=2^{-j}(j \geq 0)$, we have

$$
\begin{aligned}
& \int_{0}^{1}(1-r)^{\frac{1}{q}-2}\left(\sum_{j=1}^{\infty} \frac{\omega\left(\delta_{j}\right)}{\left(1+\delta_{j}-r\right)^{\frac{1}{p}-1}}\right) d r=\sum_{j=1}^{\infty} \omega\left(\delta_{j}\right) \int_{0}^{1} \frac{(1-r)^{\frac{1}{q}-2}}{\left(1+\delta_{j}-r\right)^{\frac{1}{p}-1}} d r \\
\geq & \sum_{j=1}^{\infty} \omega\left(\delta_{j}\right) \int_{1-\delta_{j}}^{1-\delta_{j+1}} \frac{(1-r)^{\frac{1}{q}-2}}{\left(1+\delta_{j}-r\right)^{\frac{1}{p}-1}} d r \approx \sum_{j=1}^{\infty} \delta_{j}^{\frac{1}{q}-2} \omega\left(\delta_{j}\right) \int_{1-\delta_{j}}^{1-\delta_{j+1}} \frac{1}{\left(1+\delta_{j}-r\right)^{\frac{1}{p}-1}} d r .
\end{aligned}
$$

A simple calculation shows that

$$
\int_{1-\delta_{j}}^{1-\delta_{j+1}} \frac{1}{\left(1+\delta_{j}-r\right)^{\frac{1}{p}-1}} d r=K \delta_{j}^{2-\frac{1}{p}}, \quad j \geq 1,
$$

and then (27) and (26) yield

$$
\sum_{j=1}^{\infty} \frac{\omega\left(\delta_{j}\right)}{\delta_{j}^{\frac{1}{p}-\frac{1}{q}}}<\infty
$$

It is easy to see that this implies that

$$
\int_{0}^{1} \frac{\omega(t)}{t^{1+\frac{1}{p}-\frac{1}{q}}} d t<\infty
$$

and this is equivalent to

$$
\int_{0}^{1} \frac{\phi(r)}{(1-r)^{\frac{1}{p}-\frac{1}{q}}} d r<\infty
$$


Acknowledgements The authors wish to thank the referee for his/her useful comments.

The first author is partially supported by an MCyT grant BMF2002-04013, Spain. The second and third authors are partially supported by a grant from "La Junta de Andalucía" (FQM-210) and by grants from the spanish government (BFM20011736 and MTN2004-00078).

\section{References}

[1] J.M. Anderson, J. Clunie and Ch. Pommerenke, On Bloch functions and normal functions, J. Reine Angew. Math. 270 (1974), 12-37.

[2] O. Blasco, Operators on weighted Bergman spaces $(0<p \leq 1)$ and applications, Duke Math. J. 66 (1992), $443-467$.

[3] O. Blasco, Multipliers on weighted Besov spaces of analytic functions, Contemp. Math. 144 (1993), 23-33.

[4] O. Blasco, D. Girela and M.A. Márquez, Mean growth of the derivative of analytic functions, bounded mean oscillation, and normal functions, Indiana Univ. Math. J. 47, (1998) 893-912.

[5] O. Blasco and G. Soares de Souza, Spaces of analytic functions on the disc where the growth of $M_{p}(F, r)$ depends on a weight, J. Math. Anal. Appl. 147 (1990), 580-598.

[6] S. Bloom and G. Soares de Souza, Weighted Lipschitz spaces and their analytic characterizations, Constr. Approx. 10 (1994), 339-376.

[7] P.L. Duren, B.W. Romberg and A.L. Shields, Linear functionals on $H^{p}$ spaces with $0<p<1$, J. Reine Angew. Math. 238 (1969), 32-60.

[8] P.L. Duren, Theory of $H^{p}$ Spaces, Academic Press, New York-London 1970. Reprint: Dover, Mineola, New York, 2000.

[9] J. B. Garnett, Bounded Analytic Functions, Academic Press, New York, etc., 1981.

[10] D. Girela, Mean Lipschitz spaces and bounded mean oscillation, Illinois J. Math. 41 (1997), 214-230.

[11] G.H. Hardy and J.E. Littlewood, Some properties of fractional integrals. II, Math. Z. 34 (1932), 403-439.

[12] O. Lehto and K. I. Virtanen, Boundary behaviour and normal meromorphic functions, Acta Math. 97 (1957), 47-65.

[13] G.D. Levshina, Coefficient multipliers of Lipschitz functions (Russian), Mat. Zametki 52 (1992), 68-77; english transl. in Math. Notes 52 (1993), 1124-1130.

[14] J. E. Littlewood and R. E. A. C. Paley, Theorems on Fourier series and power series. II, Proc. London Math. Soc. 42 (1936), 52-89.

[15] D. H. Luecking, A new proof of an inequality of Littlewood and Paley, Proc. Amer. Math. Soc. 103 (1988), $887-893$.

[16] S.A. Vinogradov, Multiplication and division in the space of analytic functions with area integrable derivative, and in some related spaces (Russian), Zap. Nauchn. Sem. S.-Peterburg. Otdel. Mat. Inst. Steklov. (POMI) 222 (1995), Issled. po Linein. Oper. i Teor. Funktsii 23, 45-77, 308; translation in J. Math. Sci. (New York) 87, no. 5 (1997), 3806-3827.

[17] S. Yamashita, A non-normal function whose derivative is of Hardy class $H^{p}, 0<p<1$, Canad. Math. Bull. 23 (1980), 499-500

[18] A. Zygmund, Trigonometric series, Vol. I and II, Second ed., Cambridge Univ. Press, London and New York, 1959. 